\title{
KamLAND: geo-neutrino measurement in Japan
}

\author{
Itaru Shimizu
}

Research Center for Neutrino Science, Tohoku University, Sendai, Japan

\author{
Article history \\ Received July 5, 2016; accepted October 5, 2016. \\ Subject classification: \\ Geo-neutrino measurement, Anti-neutrino detector, KamLAND, Japan.
}

\begin{abstract}
Geoneutrinos are antineutrinos produced in radioactive isotope decays inside the Earth. Those antineutrinos are neutral particle and can be detected by weak interaction, however, due to extremely small cross section, there were no feasible experiments for a long time. The observation of geoneutrinos has been finally made using large-size liquid scintillator detectors, KamLAND and Borexino. In this article, the latest result of KamLAND and future prospects of geo $\bar{v}_{\mathrm{e}}$ measurements are reviewed.
\end{abstract}

\section{Introduction}

Electron antineutrinos $\left(\bar{v}_{\mathrm{e}}\right)$ emitted by radioactivities within Earth's interior is called geoneutrinos. They are mainly produced by beta-decays of primordial radioactivities, ${ }^{238} \mathrm{U},{ }^{232} \mathrm{Th}$, and ${ }^{40} \mathrm{~K}$, and have energies below 3.4MeV. Such series of decays also produce radiogenic heat, which primarily contributes to Earth's energetics. Therefore, we can estimate Earth's heat production through the measurement of geoneutrino flux, and make a comparison with the surface heat flow, resulting in an important input for geophysics. Geoneutrino detection provides a unique tool to investigate Earth's interior, however, it used to be regarded as almost infeasible for a long time, due to extremely small cross section of the neutrino interaction. In the late 1990s, plans of feasible experiments to detect geo $\bar{v}_{\mathrm{e}}$ 's, KamLAND in Japan and Borexino in Italy, are suggested for the first time. In this article, the latest result of KamLAND and future prospects of geo $\bar{v}_{\mathrm{e}}$ measurements are reviewed.

\section{KamLAND experiment}

KamLAND (Kamioka liquid scintillator anti-neutrino detector) is located in Kamioka mine, Japan, at a depth of $1000 \mathrm{~m}$ to suppress the cosmic-ray muon backgrounds. The detector consists of 1000 ton of ultra-pure liquid scintillator (LS) contained in a transparent nylonbased balloon suspended in nonscintillating oil. The containment balloon is a 13 -m-diameter spherical bal- loon made of 135 - $\mu$ m-thick transparent nylon/EVOH composite film, and is suspended with Kevlar ropes. The neutrino interaction produces energetic charged particle in the LS, and a LS excitation by its energy loss results in a fluorescence emission (scintillation light). This scintillation light is viewed by photomultiplier tubes (PMTs), an array of 1879 PMTs, mounted on the inner surface of the containment vessel. To recored the signals from PMTs, KamLAND has an electronics system called Front-End Electronics, based on an analog transient waveform digitizer (ATWD). When a PMT channel registers a "hit", the charge as a function of time is recorded by an ATWD chip. Each channel has two sets of three ATWDs, which record waveforms at three available gain levels, allow us to cover signals from wide dynamic range.

\section{Geo-neutrino results}

In the KamLAND geoneutrino measurement, the

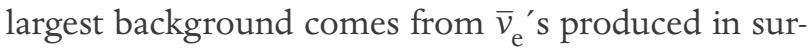
rounding commercial nuclear reactors in Japan. The expected reactor $\bar{v}_{\mathrm{e}}$ flux is well calculated by neutrino oscillation parameters which are determined from KamLAND spectral data, as well as the nuclear fission rate based on the reactor operation records. It indicates the geoneutrino contribution can be extracted based on a statistical subtraction of reactor $\bar{v}_{\mathrm{e}}$ backgrounds. In 2005, KamLAND showed the first experimental study of geo $\bar{v}_{\mathrm{e}}$ 's [Araki et al. 2005], and the observed flux was consistent with Earth model expectations. It was followed by an improved geo $\bar{v}_{\mathrm{e}}$ measurement owing to statistical increase and radioactive background decrease by a factor of $\sim 20$ after the LS purification [Gando et al. 2011]. After the Fukushima nuclear accident in March 2011, the reactor $\bar{v}_{\mathrm{e}}$ rate significantly decreased because of the long-term shutdown of Japanese reactors in a review of nuclear safety standard. This reactor-off situation provided a good data for the geoneutrino observation. 


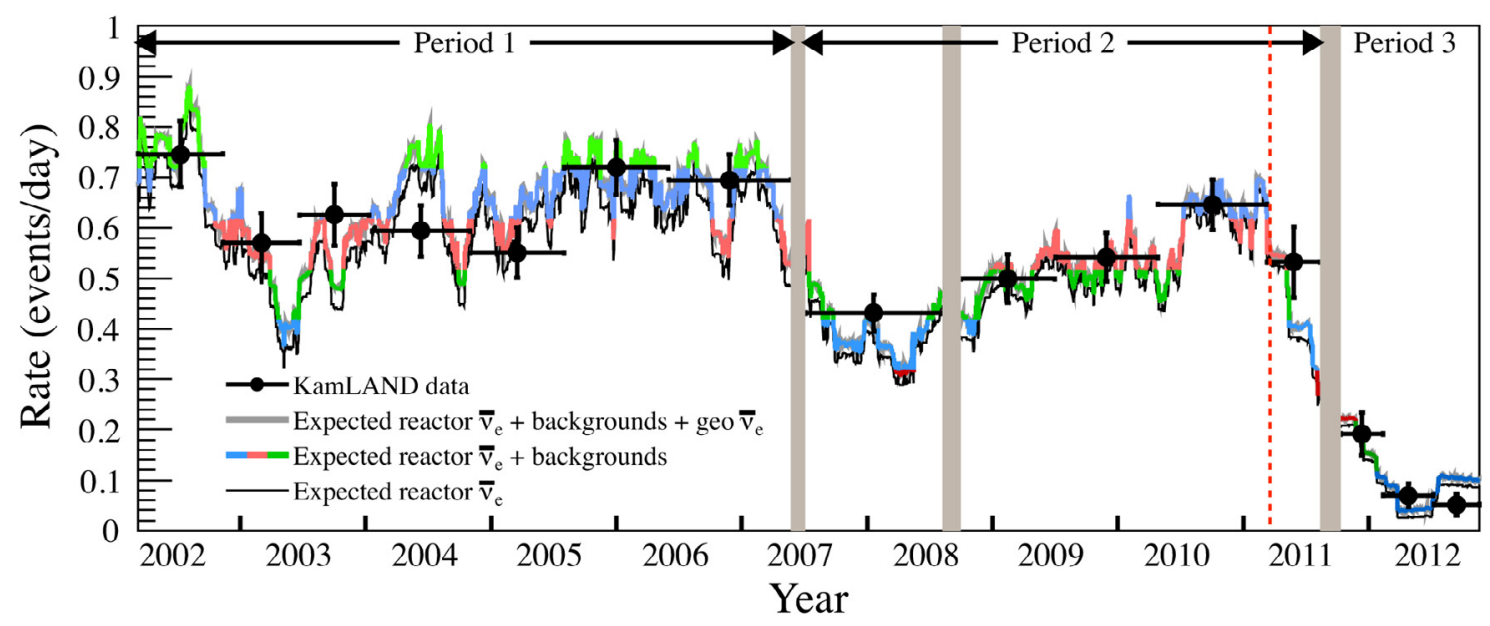

Figure 1. Time variation of expected and observed rates of $\bar{v}_{\mathrm{e}}$ 's with energies above 3.4MeV in KamLAND data over 10 years [Gando et al. 2013]. The vertical grey bands indicate deadtime during the liquid scintillator purification and the detector modification. The significant reduction of $\bar{v}_{\mathrm{e}}$ flux from reactors after March 2011 earthquake (red dashed line) is remarkable, and provides a new opportunity of the "Reactor on-off" study for neutrino oscillations and geo $\bar{v}_{\mathrm{e}}$ 's below $3.4 \mathrm{MeV}$.

The latest KamLAND result [Gando et al. 2013] is based on a total livetime of 8.19 years. The data set is divided into three periods: Period 1 (March 2002 - May 2007); before the LS purification, Period 2 (May 2007 August 2011); during and after the LS purification, and Period 3 (October 2011 - November 2012); after the start of the KamLAND-Zen neutrino-less double betadecay experiment. Figure 1 shows the time variation of the observed rate of $\bar{v}_{\mathrm{e}}$ 's with energies above $3.4 \mathrm{MeV}$ in agreement with the expectations of reactor $\bar{v}_{\mathrm{e}}$ 's. The $\bar{v}_{\mathrm{e}}$ rate in Period 3 is remarkably smaller than other periods. To exploit the time variation of the known back-

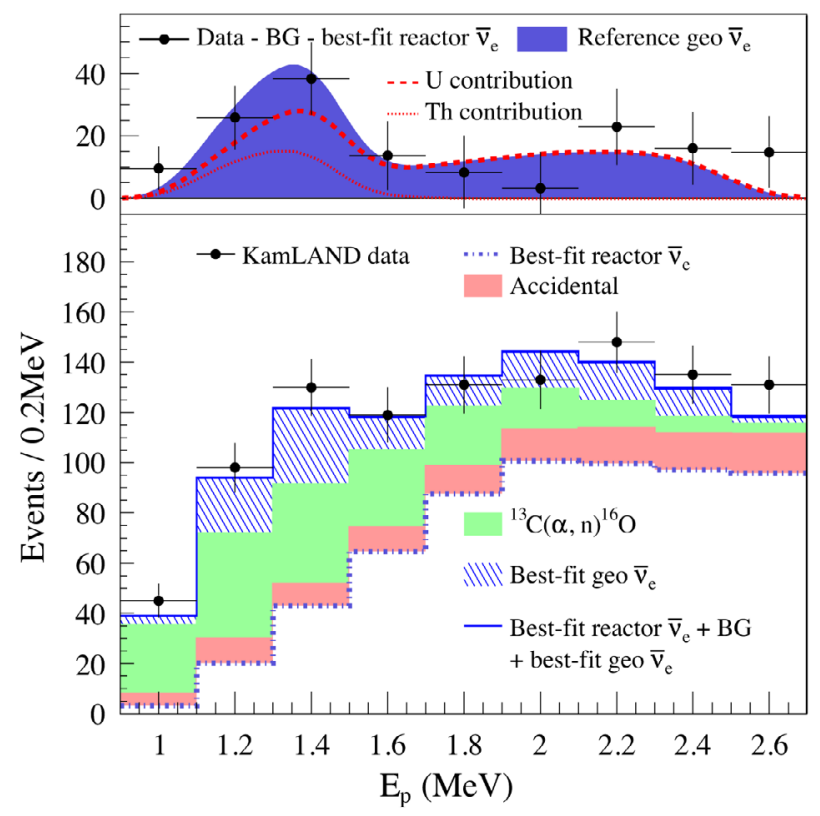

Figure 2. Energy spectrum of the KamLAND observed events [Gando et al. 2013]. The best-fit contributions are indicated by colored histograms (bottom panel), and the spectrum after subtracting the non-geo-neutrino contributions is compared with the Earth model prediction (top panel). ground rate, $\bar{v}_{\mathrm{e}}$ candidates are analyzed with an unbinned maximum-likelihood method incorporating the event rate and the prompt energy, including the event time.

The KamLAND $\bar{v}_{\mathrm{e}}$ analysis constrains both the geo $\bar{v}_{\mathrm{e}}$ rates and the neutrino oscillation parameters simultaneously. As discussed in Gando et al. [2013], the precise measurement of neutrino oscillation parameters was achieved in KamLAND; $\tan ^{2} \theta_{12}=0.436_{-0.025}^{+0.029}$, $\Delta m_{21}^{2}=7.53_{-0.18}^{+0.18} \times 10^{-5} \mathrm{eV}^{2}$, and $\sin ^{2} \theta_{13}=0.023_{-0.002}^{+0.002}$, incorporating constraints from solar neutrino, accelerator, and short-baseline reactor neutrino experiments. The energy spectrum of the observed events together with the best-fit contributions in the energy region of geo $\bar{v}_{\mathrm{e}}$ 's is shown in Figure 2. The main background contributors are the reactor $\overline{\mathrm{e}}_{\mathrm{e}},{ }^{13} \mathrm{C}(\alpha, n){ }^{16} \mathrm{O}$ reactions, and accidental coincidence events in descending order. In Period 2 and later, the background from ${ }^{13} \mathrm{C}(\alpha, n){ }^{16} \mathrm{O}$ decreased due to the LS purification, and in Period 3, the reactor $\bar{v}_{\mathrm{e}}$ background decreased. It results in the significant low background condition in Period 3. We find the clear event excess in the backgroundsubtracted spectrum as shown Figure 2 (top panel), and the background-subtracted rate and spectral shape agree with Earth model expectations for each data set. The best-fit geo $\bar{v}_{\mathrm{e}}$ 's are 116 and 8 events from $U$ and Th decays, respectively, with confidence level (C.L.) contours as shown in Figure 3. Assuming the Th/ $\mathrm{U}$ mass ratio of 3.9 based on the geochemical model [McDonough and Sun 1995], the total number of geo $\bar{\nu}_{\mathrm{e}}$ events is $116_{-27}^{+28}$, which corresponds to an oscillated $\bar{v}_{\mathrm{e}}$ flux of $3.4_{-0.8}^{+0.8} \times 10^{6} \mathrm{~cm}^{-2} \mathrm{~s}^{-1}$ at KamLAND.

\section{Earth model comparison}

The result of geo $\bar{v}_{\mathrm{e}}$ flux measurements in KamLAND will provide a new constraint on both geophysics 
and geochemistry. Firstly, we calculate the geo $\bar{v}_{\mathrm{e}}$ flux at Kamioka for different bulk silicate Earth (BSE) compositional estimates based on simple but appropriate assumption, to compare with the KamLAND geo $\bar{v}_{\mathrm{e}}$ data. The crustal contribution for $U$ and Th can be estimated by compositional data through rock sampling. On the other hand, the chemical composition of the mantle, most massive layer of Earth's interior, is uncertain. So we estimate the total amount of $U$ and Th, predicted by the BSE models, and assume that the excess above the crustal contribution uniformly distribute in the mantle [Enomoto et al. 2007]. The KamLAND data tested three BSE compositional estimates of geo $\bar{v}_{\mathrm{e}}$ flux and radiogenic heat from ${ }^{238} \mathrm{U}$ and ${ }^{232} \mathrm{Th}$ [Šrámek et al. 2013], as shown in Figure 4. The uncertainties from the abundances and the distributions of $U$ and $T h$ in the mantle are indicated by the vertical bands. The upper solid line with a steeper slope corresponds to the estimate in the homogeneous mantle assumption, and the lower solid line the estimate in the sunken-layer which assumes that all of the $U$ and Th below the crust are concentrated at the mantle-core boundary. While the statistical treatment of geological uncertainties is not straightforward, assuming Gaussian errors for the crustal contribution and for the BSE abundances, the KamLAND data disfavor the geodynamical models with the homogeneous hypothesis at $89 \%$ C.L. However, due to the limited statistical power of the data, all BSE composition models are still consistent within $\sim 2 \sigma$ C.L. The measured geo $\bar{v}_{\mathrm{e}}$ flux can be converted into a total radiogenic heat of $11.2_{-5.1}^{+7.9} \mathrm{TW}$ from $\mathrm{U}$ and Th (additional $\sim 3.0 \mathrm{TW}$ is expected from other radioactive nuclei).

After the publication of the KamLAND result in Gando et al. [2013], new reference models with refined crustal compositions become available in the literature. If the reference model [Enomoto et al. 2007] used in the analysis in Gando et al. [2013] is replaced with a modern model [Huang et al. 2013], the radiogenic heat is estimated to be $14.9_{-8.3}^{+9.8} \mathrm{TW}$ from $\mathrm{U}$ and $\mathrm{Th}$. The increase of the radiogenic heat estimation is mainly due to the change in the crustal geo $\bar{v}_{\mathrm{e}}$ flux contribution, which is the offset of the flux-heat conversion function as shown in Figure 4. It indicates, in addition to the determination of the geo $\bar{v}_{\mathrm{e}}$ flux, a precise estimate of the crustal contribution is of critical importance in proving the radiogenic heat in the mantle. In the future, the estimation needs to be further improved by the revision of the regional crustal compositions around Japan, compiling the latest knowledge in geophysics, geochemistry, and local geology. The calculations based on the different Earth models support that the total radiogenic heat is smaller than the heat flow of $47 \pm 2$ TW from Earth's surface [Davies and Davies 2010], indicating secular cooling of the Earth.

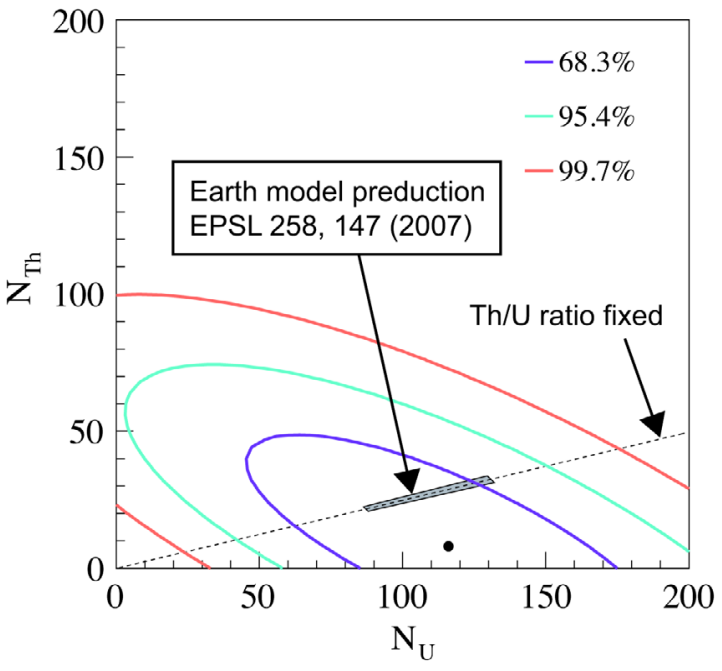

Figure 3. Confidence level (C.L.) contours for the observed number of geo $\bar{v}_{\mathrm{e}}$ events in $\mathrm{U}$ and $\mathrm{Th}$. The small shaded region represents the prediction from the reference model of Enomoto et al. [2007].

\section{Future prospect}

Currently, the geoneutrino measurements by two large LS detectors are available; KamLAND in Japan and Borexino in Italy [Bellini et al. 2013]. In the future, there are several plans of geoneutrino detectors at different locations; the $\mathrm{SNO}+$ detector close to completing the construction in Canada [Chen 2006], the JUNO detector in China [Han et al. 2016], the LENA detector in Europe [Wurm et al. 2012], and the movable Hanohano detector in a deep ocean [Learned et al. 2008]. Those detectors will have LS mass larger than KamLAND, so we expect the reduction of the statistical uncertainties on the measured $\bar{v}_{\mathrm{e}}$ flux. At the KamLAND location,

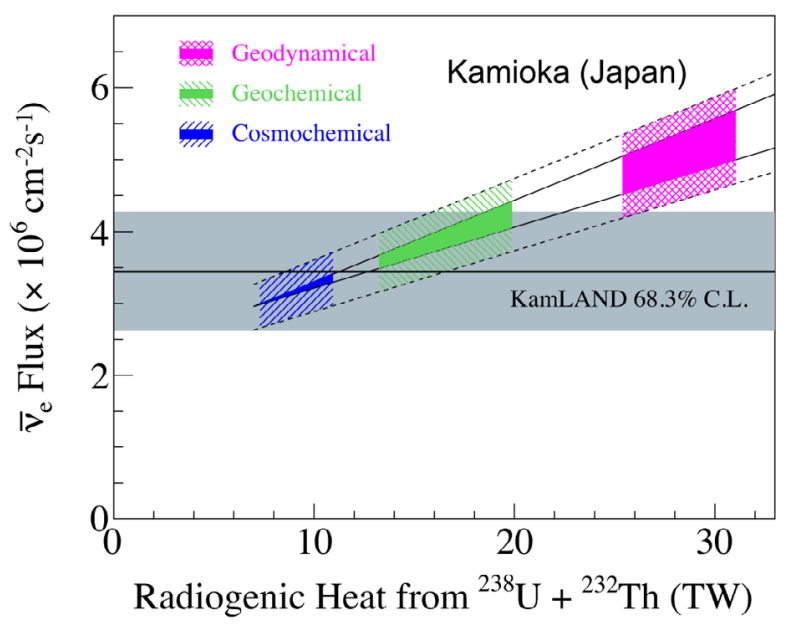

Figure 4. Three BSE compositional estimates of geo $\bar{v}_{\mathrm{e}}$ flux and radiogenic heat from ${ }^{238} \mathrm{U}$ and ${ }^{232} \mathrm{Th}$ [Gando et al. 2013]. The colored shaded regions indicate the different mantle model predictions from cosmochemical, geochemical, and geodynamical estimates, and are compared with the KamLAND measurement (gray band). The variations due to the radiochemical distributions are represented by the two solid lines with different slopes; the homogeneous and sunkenlayer hypotheses. The variations after adding the uncertainty in the crustal contribution are indicated by the two dashed lines. 
the geo $\bar{v}_{\mathrm{e}}$ contribution from the mantle is only about one fourth of the total geo $\bar{v}_{\mathrm{e}}$ flux, and may be estimated based on the subtraction of the crustal contribution depending on the crustal model. On the other hand, a detector in an oceanic location (e.g. Hanohano) has less exposure to geo $\bar{v}_{\mathrm{e}}$ 's from thick continental crusts, and will be more appropriate to measure the mantle flux. Such multi-site data at different geological locations will be useful to construct a detail map of neutrino sources inside the Earth. In addition, detectors distant from commercial reactors will reduce the uncertainties on the measured geo $\bar{v}_{\mathrm{e}}$ flux.

Alternative approach to mapping neutrino sources is the directional measurement of $\bar{v}_{\mathrm{e}}$ 's using a detector with a sensitivity of coming $\bar{v}_{\mathrm{e}}$ direction [Shimizu 2007]. Such measurements will provide the information on separate contributions from the crust and mantle even at locations near continental crusts, based on the nadir angle distribution. A ${ }^{6} \mathrm{Li}-\mathrm{loaded}$ detector with relatively good angular resolution can be a candidate, and the possibility of applications for exploring hypothetical magmatic reservoirs was studied in Tanaka and Watanabe [2014]. They are new experimental techniques imaging Earth's interior using neutrinos. Further development of the detector performance and application researches are anticipated in the future.

\section{Conclusion}

The KamLAND data collected between March 2002 and November 2012 provides a most precise measurement of the geoneutrino flux. The observed energy spectra are consistent with the expectation of ${ }^{238} \mathrm{U}$ and ${ }^{232} \mathrm{Th}$ decays in the Earth. Based on an appropriate geophysical assumption, the observed geoneutrino flux is in agreement with the predictions from three BSE models within $\sim 2 \sigma$ C.L. Currently, due to the limited statistical power, the clear discrimination between models has not been achieved yet. In addition, the crustal contribution needs to be estimated more precisely based on the latest knowledge in geophysics, geochemistry, and local geology. In the future, if multi-site measurements with larger statistics at different geological locations become available, a detail map of neutrino sources inside the Earth can be constructed. The directional measurement of $\bar{v}_{\mathrm{e}}$ can be an alternative experimental technique imaging Earth's interior.

\section{References}

Araki, T., et al. (KamLAND collaboration) (2005). Experimental investigation of geologically produced antineutrinos with KamLAND, Nature, 436, 499-503.

Bellini, G., et al. (Borexino collaboration) (2013). Measurement of geo-neutrinos from 1353 days of Borex- ino, Phys. Lett. B, 722, 295-300.

Chen, M.C. (2006). Geo-neutrinos in SNO+, Earth Moon Planets, 99, 221-228.

Davies, J.H., and D.R. Davies (2010). Earth's surface heat flux, Solid Earth, 1, 5-24.

Enomoto, S., E. Ohtani, K. Inoue, and A. Suzuki (2007). Neutrino geophysics with KamLAND and future prospects, Earth Planet. Sci. Lett., 258, 147-159.

Gando, A., et al. (KamLAND collaboration) (2011). Partial radiogenic heat model for Earth revealed by geoneutrino measurements, Nat. Geosci., 4, 647-651.

Gando, A., et al. (KamLAND collaboration) (2013). Reactor on-off antineutrino measurement with KamLAND, Phys. Rev. D, 88, 033001.

Han, R., Y.-F. Li, L. Zhan, W.F. McDonough, J. Cao and L. Ludhova (2016). Potential of geoneutrino measurements at JUNO, Chinese Phys. C, 40, 033003.

Huang, Y., V. Chubakov, F. Mantovani, R. Rudnick and W.F. McDonough (2013). A reference Earth model for the heat-producing elements and associated geoneutrino flux, Geochem. Geophy. Geosy., 14, 2003-2029.

Learned, J.G., S.T. Dye and S. Pakvasa (2008). Hanohano: A Deep Ocean Anti-Neutrino Detector for Unique Neutrino Physics and Geophysics Studies, In: Proceedings of the Twelfth International Workshop on Neutrino Telescopes (Venice, March 2007), arXiv: 0810.4975 .

McDonough, W.F., and S.-s. Sun (1995). The composition of the Earth, Chem. Geol., 120, 223-253.

Shimizu, I. (2007). Directional measurement of antineutrinos, Nucl. Phys. B - Proc. Supp., 168, 147-149.

Šrámek, O., W.F. McDonough, E.S. Kite, V. Lekic, S.T. Dye and S. Zhong (2013). Geophysical and geochemical constraints on geoneutrino fluxes from Earth's mantle, Earth Planet. Sci. Lett., 361, 356-366.

Tanaka, H.K.M., and H. Watanabe (2014). ${ }^{6}$ Li-loaded directionally sensitive anti-neutrino detector for possible geo-neutrinographic imaging applications, Sci. Rep.-UK, 4, 4708 EP.

Wurm, M., et al. (2012). The next generation liquidscintillator neutrino observatory LENA, Astropart. Phys., 35, 685-732.

Corresponding author: Itaru Shimizu,

Research Center for Neutrino Science, Tohoku University,

Sendai, Japan; email: shimizu@awa.tohoku.ac.jp.

(C) 2017 by the Istituto Nazionale di Geofisica e Vulcanologia. All rights reserved. 